\title{
TYPE 1 DIABETES MELLITUS
}

Moreno Moreno, P; Palomares Ortega, RJ; Muñoz Jiménez, C; Gálvez Moreno, MA

Management Unit of Clinical Endocrinology and Nutrition. University Hospital Reina Sofia. Córdoba. Spain.

\section{OBJECTIVE}

The aim of this study was to evaluate maternal and neonatal outcomes in women with Type 1 Diabetes Mellitus (DM-1), and analyzing differences associated to insulin glargine exposure during pregnancy.

\section{PATIENTS AND METHODS}

Retrospective descriptive study of pregnancies in women with DM-1 (2004-2012). Variables analyzed: baseline characteristics (age, time of diabetes evolution, microvascular complications, weight), maternal outcomes (weight gain, HbA1c, hypoglycemia, preeclampsia, abortions, vaginal delivery and cesarean section) and neonatal outcomes (gestational age at delivery, birth weight and congenital malformations). The pregnancies were divided in groups attending to their exposition to glargine insulin and were analyzed to evaluate possible differences between them (group 1: exposed, group 2: unexposed). Statistical analysis: comparing proportions with the chisquared and comparing means with Student's test.

\section{RESULTS}

132 pregnancies in women with DM-1

Group 1: 43.2\%, group 2: $56.8 \%$.

Baseline characteristics were similar in both groups

\begin{tabular}{|c|c|c|c|}
\hline HbA1c (\%) & Group 1 & & Group 2 \\
\hline Before pregnancy & 7,95 & $p=0,001$ & 7,02 \\
\hline First trimester & 6,83 & $p=0,34$ & 6,47 \\
\hline Second trimester & 6,45 & $p=0,92$ & 6,33 \\
\hline Third trimester & 6,67 & $\mathrm{p}=0,88$ & 6,42 \\
\hline
\end{tabular}

Maternal weight gain was similar: Group 1 vs group 2: $10,28 \mathrm{Kg}$ vs $11,02 \mathrm{Kg}(\mathrm{p}=0,4)$

\section{CONCLUSIONS}

The number of caesarean sections was higher in women not exposed to insulin glargine. Insulin glargine use before and during pregnancy is not associated with worse maternal and neonatal outcome, or congenital malformation.

\begin{tabular}{|c|c|c|c|}
\hline $\begin{array}{l}\text { Maternal } \\
\text { outcomes }\end{array}$ & \multicolumn{2}{|c|}{ Group 1} & Group 2 \\
\hline Abortions (\%) & 2 & $p=0,05$ & 12 \\
\hline Caesarean section (\%) & 31,5 & $p=0,005$ & 52 \\
\hline $\begin{array}{c}\text { Gestational age at } \\
\text { delivery (week) }\end{array}$ & 38,02 & $p=0,92$ & 38,12 \\
\hline
\end{tabular}

\begin{tabular}{|c|c|c|c|}
\hline $\begin{array}{l}\text { Neonatal } \\
\text { outcomes }\end{array}$ & Group 1 & & Group 2 \\
\hline Macrosomia (\%) & 23 & $p=0,29$ & 18 \\
\hline $\begin{array}{c}\text { Congenital } \\
\text { malformations (\%) }\end{array}$ & 23 & $p=0,49$ & 28 \\
\hline Hipoglycemia (\%) & 2 & $p=0,13$ & 7 \\
\hline
\end{tabular}

